\title{
Rufus H. Gouws, Ulrich Heid, Wolfgang Schweickard and Herbert Ernst Wiegand (eds.) Dictionaries. An International Encyclopedia of Lexicography. Supplementary Volume: Recent Developments with Focus on Electronic and Computational Lexicography (HSK Vol. 5.4). Berlin: De Gruyter Mouton, 2013. XIII + 1,579 pp. € 499, US \$ 699 ISBN 978-3-11-023812-9
}

\section{Reinhard Hartmann}

Published online: 24 February 2015

(C) Springer Berlin Heidelberg 2015

A welcome arrival is the follow-up volume 5.4 of the authoritative encyclopedia Wörterbücher/Dictionaries/Dictionnaires which was first published in three volumes (5.1 in 1989, 5.2 in 1990 and 5.3 in 1991) as part of the international series Handbücher zur Sprach- und Kommunikationswissenschaft, of which 38 titles have appeared so far on subjects like linguistics, semantics, LSP, terminology and translation. ${ }^{1}$

How does this encyclopedia of lexicography manage to deal with our complicated field - not only to supplement the three volumes published more than 20 years ago, but also to report on recent developments in computing? The task was taken on by scholars well-known for their achievements in promoting the study of lexicography, but as we shall see, this was quite a difficult task to achieve.

The material is presented in the form of articles, which are subdivided into numbered sections and sub-sections, each with an outline and a list of bibliographical references and many with numbered dictionary "extracts", "entries", "definitions" and "examples" as well as illustrative figures and tables (although their marking with these titles is not always consistent). The 110 articles are grouped into 20 chapters (see below).

The Preface provides the historical background from 1989-91 to 2012 and outlines the contents of this supplementary volume, which must have been an ever-

\footnotetext{
1 This volume and some others are available from Walter de Gruyter GmbH as ebooks: see http://www. degruyter.com/browse? $\mathrm{t} 1=\mathrm{SK}-12-01$.
}

R. Hartmann $(\square)$

40 Velwell Road, Exeter, UK

e-mail: R.R.K.Hartmann@exeter.ac.uk 
growing and overpowering project, as it has involved 110 authors from 21 different countries. There is no direct relation between the identical number (110) of articles and authors, as some have collaborated with others and several have supplied more than one article. ${ }^{2}$ These relationships must have provided complex and at times even irritable editorial duties, as most authors had to be given intensive guidance and allowed mutual concessions. Some of these problems are mentioned by the editors in the Preface, such as their decision this time to offer the text of the whole book through the medium of English. The help of a range of editorial assistants, proofreaders, translators, illustrators and indexers is also acknowledged by them. All this must have meant considerable challenges, and does explain the long delay in production of the volume.

As lexicography is still a relatively young academic discipline, it must have been extremely difficult for the editors to decide which aspects to concentrate on, how to arrange them in groups of chapters, and which to ignore. The main topics selected are:

1. New developments in lexicographic theory (or "metalexicography"), Articles 1-36 in Chapters I to VI;

2. New developments in the dictionary-making process representing various languages, Articles 37-64 in Chapters VII to XII; and

3. New developments in computational lexicography, Articles 65-110 in Chapters $\mathrm{XIII}$ to $\mathrm{XX}$.

A full list of the 20 chapters is available to readers of this review in the form of an informative table of contents, together with an outline of each of the 110 articles as well as extracts of them, on the publisher's website. ${ }^{3}$

The first group of topics (Chapters I to VI) includes two introductory articles, by the editors themselves, on dictionaries in modern society and on the impact of computational techniques. The latter have not only grown profusely as part of NLP, since the first three volumes, but have also been applied in a number of ways, for e.g. corpus collection and for developing new types of electronic dictionaries for human use. This is followed by five chapters on the relevance of lexicographic theory and management. One of these (Article 3 in Chapter II) stands out as an important overview of the various ways in which information is selected and located, searched and found in dictionaries, in terms of such underlying features as "macrostructures", "access structures", "microstructures" and "mediostructures". How all this affects the textual architecture of dictionary entries is discussed in Articles 4-10 in Chapter II, exploring the ways in which the organizational complexity of dictionaries can be represented. Unfortunately, readers may find this survey rather hard to absorb as this topic is inherently enigmatic, complicated by many theoretical innovations and terminological ambiguities as well as by literal

\footnotetext{
${ }^{2}$ I have myself contributed 3 articles to Volumes 5.1 and 5.3 and another 3 to Volume 5.4.

3 http://www.degruyter.com/viewbooktoc/product/175228.
} 
(German-to-English) translations and abstract diagrammatic illustrations (some based on models borrowed from mathematical set theory).

These structure-oriented contributions are followed by 9 articles in Chapter III on "dictionary typology", including useful descriptions of various general, learner's and special-purpose as well as electronic dictionaries, the treatment of selected dictionary subjects such as collocations and culture-bound items (6 articles in Chapter IV), research on dictionary production and use (6 articles in Chapter V), and lexicographic training, dictionary criticism and metalexicographic organization (5 articles in Chapter VI).

The second group of topics (Chapters VII to XII) traces the lexicographic traditions in various language families, ranging from articles on the ancient languages of the Near East and Greek and Latin to articles on Romance, Germanic and Slavic languages, on Arabic, Chinese, Japanese and Korean, and on languages in Africa. They constitute very useful although rather diverse summaries, some of which report on massive developments since their treatment in Volumes 5.1-5.3. For example, Article 49 on German synchronic lexicography gives the most impressive description of more than 200 dictionaries, supported by useful illustrations and comparisons (other languages such as English, French and Chinese are not as well documented).

The third group of topics (Chapters XIII to XX) begins with the history of computational lexicography and corpus linguistics, then treats the typology of electronic dictionaries (divided between articles on interactive or "human use", including internet dictionaries, dictionary portals, pedagogical dictionaries and electronic encyclopedias, and articles on NLP or "machine use", including speech recognition and synthesis, text mining, database searches and machine-aided translation). Further articles discuss models for the representation of dictionaries (the "form aspect", including formalisms for electronic dictionary resources); models for the representation of linguistic data (the "content aspect", including codes for syntactic and semantic properties); computer-based dictionary-making with the aid of corpora and other tools; and finally, the computational aspects of terminology and terminography.

Most apparent and some unresolved issues raised in the original three volumes are addressed here, including tensions between human and machine use, lexicographic compilation practice and metalexicographic theory, and production versus use, for e.g. the "user perspective". Many disciplinary and terminological questions are answered, for e.g. what terms might be appropriate to designate our (widened) field, such as "reference science". All the material in this encyclopedia is well collected, coordinated and presented, supported by the two indexes of subjects and names. After examining it in detail, I conclude that this book is a valuable addition to any library of essential sources of knowledge on the subject of lexicography.

\footnotetext{
${ }^{4}$ The User in Focus is the sub-title of the Proceedings of the XVI EURALEX International Congress, which has recently been made available to readers anywhere, allowing them to download the content of the whole volume from http://www.euralex.org/proceedings-toc/euralex_2014/. A significant proportion of the papers were actually assessed by a panel of experts who had been editors or authors of the Dictionaries volume reviewed here.
} 